\title{
ECTD Promotional Material Audience Type Terminology
}

National Cancer Institute

\section{Source}

National Cancer Institute. eCT D Promotional Material Audience Type Terminology. NCI Thesaurus. Code C97188.

Terminology developed to support eCT D Promotional Promotional Material Audience Types. 